\title{
A DISTRIBUTED DAMAGE APPROACH TO COMBINED BENDING AND AXIAL LOADING OF ROCK BEAMS
}

\author{
F. Ouchterlony \\ Swedish Detonic Research Foundation, SueDeFo, P.O. Box 32058, \\ S-12611 Stockholm, Sweden
}

\begin{abstract}
The predictive capabilities of continuum damage mechanics (CDM) as to eccentric uniaxial loading and pure bending of strength test specimens of rock are shown. For eccentric loading much smaller strength reductions than usual are predicted, for bending rupture factors that depend properly on section shape. Experimental results support these predictions.
\end{abstract}

KEYWORDS

Continuum damage mechanics; non-linear material behavior; rock mechanics; tensile strength testing; eccentric loading; strain gauges; rupture factor.

\section{INTRODUCTION}

Eccentric loading makes the uniaxial testing of brittle materials a difficult tensile strength test to perform satisfactorily. The conventional beam theory approach to such loading as a case of combined bending and axial stresses (Timoshenko 1940) yields a reduced apparent strength

$$
\sigma_{\text {nom }}^{f} / \sigma_{t}=1 /\left(1+e c / r_{i}^{2}\right) \text {. }
$$

Here $\sigma_{\text {nom }}^{f}$ is the nominal section stress at failure and $\sigma_{t}$ the uniaxial ten-

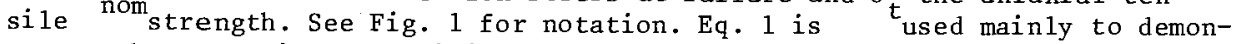
strate the necessity of avoiding eccentric loading which the uniaxial tensile testing practice for rock tries to do.

The tensile strength of rock is however mostly determined from indirect tests with resulting values that usually are much higher than $\sigma_{t}$. The apparent failure strength of a beam of brittle material in bending, $\sigma_{t b}$, is an example. In nondimensional form

$$
R=\sigma_{t b} / \sigma_{t}=\left[M_{m} /(I / c)\right] / \sigma_{t},
$$

MEM VOL2-M* 
it is called the rupture factor. $M$ is the maximum bending moment. $R$ depends on the beam material, the shape of the cross section, and the type of bending. Typical values for rock 1 ie between 1.5 and 2.5 (Vutukuri and co-workers, 1974).

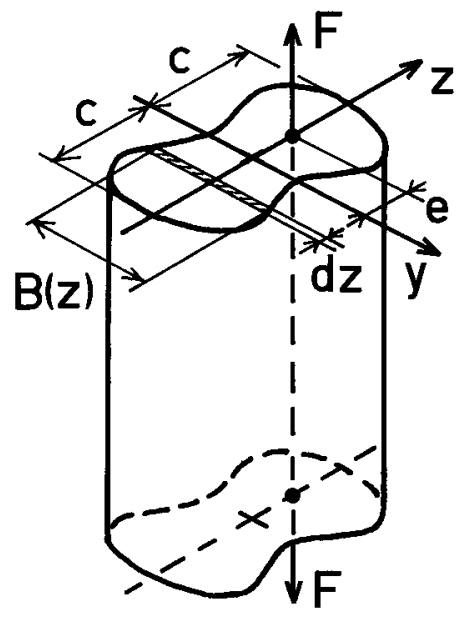

$\begin{array}{ll}\begin{array}{ll}\text { Notation } \\ \text { load }\end{array} & \mathrm{F}[\mathrm{N}] \\ \text { principal axes } & \mathrm{x}, \mathrm{y} \\ \text { bending plane } & \mathrm{z} \\ \text { breadth function } & \mathrm{B}(\mathrm{z})[\mathrm{m}] \\ \text { cross section area } & \mathrm{A}=\int \mathrm{Bdz}\left[\mathrm{m}^{2}\right] \\ \text { moment of inertia } & \mathrm{I}=\mathrm{I}=\int \mathrm{Bz}^{2} \mathrm{dz}\left[\mathrm{m}^{4}\right] \\ \text { radius of gyration } & \mathrm{r}_{i}=V(\mathrm{I} / \mathrm{A})[\mathrm{m}] \\ \text { extreme fiber position } & \mathrm{c}[\mathrm{m}] \\ \text { amount of eccentricity } & \mathrm{e}[\mathrm{m}] \\ \text { nominal section stress } & \sigma_{\mathrm{nom}}=\mathrm{F} / \mathrm{A}[\mathrm{Pa}]\end{array}$

Fig. 1 Uniaxial tensile testing of a specimen with doubly symmetric cross section of constant area, subjected to eccentric loading along one of the principal axes.

The failure criterion behind Eq. 1, that failure occurs when the tensile extreme fiber stress reaches $\sigma_{t}$, obviously can't explain why $R>1$. A new simple ap ${ }^{-}$. proach which can predict both the effects of eccentric loading in uniaxial strength testing and the variation of $R$ with section shape is continuum damage mechanics or CDM, as this contribution wi11 show. See also Ouchterlony (1981).

CDM smears out the micro-cracking or damage and it results in non-1inear material behavior, as is usually the case with rock. Other CDM work which is related to the tensile testing of brittle materials is that of Jansson (1978), Hult (1979), Krajcinovic (1979), Grady and Kipp (1981), and Krajcinovic and Fonseka (1981). A general review is given by Chaboche (1981).

\section{CDM IN UNIAXIAL TENSION}

The derivations rely on scalar CDM (Jansson, 1978). Consider the specimen in Fig. 1, subjected to uniaxial tension. Let A be the macroscopically observable section area and $A_{\text {eff }}$ the effective load carrying one. Then

$$
\mathrm{F}=\sigma \mathrm{A}=\mathrm{sA} \text { eff }
$$

define the apparent stress $\sigma$ and the net stress $s$. The amount of damage is defined by the scalar

$$
\omega=\left(A_{\text {eff }}\right) / A \text { whence } s=\sigma /(1-\omega) \text {. }
$$

Thus $\omega=0$ describes intact material and $\omega=1$ total inability to carry load. We assume that the material is 1 inearly elastic and linearly damaging. Thus 


$$
\begin{aligned}
& s=E \varepsilon \text { and } \\
& \omega=\omega_{0}+s / D \text { when } s>-\omega_{0} D \text { and } \omega=0 \text { when } s \leq-\omega_{0} D .
\end{aligned}
$$

Here $\varepsilon$ denotes the engineering strain, E the Young's modulus, D the damage modulus, and $\omega$ the initial damage. For a non-damaging material $D \rightarrow \infty$. $\omega_{0}$ represents the frequently large number of pores and micro-cracks in rock.

These equations yield $\mathrm{F}=\mathrm{s}\left(1-\omega_{\mathrm{o}}-\mathrm{s} / \mathrm{D}\right) \mathrm{A}$ expressed in net stress. The specimen will fail when it looses its 10 ad carrying capacity, that is when $\mathrm{dF} / \mathrm{d} \varepsilon \propto$ $\propto \mathrm{dF} / \mathrm{ds}=0$ and $\mathrm{F}(\mathrm{s})$ has a maximum. The resulting failure stresses become

$$
s_{t}=\frac{1}{2}\left(1-\omega_{o}\right) \mathrm{D} \text { and } \sigma_{t}=\frac{1}{4}\left(1-\omega_{0}\right)^{2} \mathrm{D} \text {, }
$$

for the damaging material. For the non-damaging material $F(s)$ is linear and a failure criterion $s=s_{t}$ has to be invoked directly.

\section{CDM IN ECCENTRIC UNIAXIAL LOADING}

Consider eccentric uniaxial loading of the specimen in Fig. 1, applied on a principal axis such that in-plane bending and axial loading results. We assume that plane cross sections remain plane and that the material is linearly elastic such that $\varepsilon(z)$ and $s(z)$ become linear. We may then write

$$
\mathrm{s}=\overline{\mathrm{s}}+\Delta \mathrm{s} \cdot \mathrm{z} / \mathrm{c},
$$

where $\bar{s}$ is the average net stress and $2 \cdot \Delta s$ its variation over the cross section. Thus $\omega(z)$ will also be linear as long as $\omega \geq 0$. The apparent stress distribution $\sigma(z)$ for a damaging material becomes

$$
\frac{\sigma}{\sigma_{t}}=2\left(\frac{\bar{s}}{s_{t}}+\frac{\Delta s}{s_{t}} \cdot \frac{z}{c}\right)\left[1-\frac{1}{2}\left(\frac{\bar{s}}{s_{t}}+\frac{\Delta s}{s_{t}} \cdot \frac{z}{c}\right)\right] .
$$

It is parabolic with $\max \sigma=\sigma_{t}$ at $z / c=\left(s_{t}-\bar{s}\right) / \Delta s$. For the non-damaging material $\sigma(z) / \sigma_{t}=s / s_{t}$ is clearly ${ }^{t} l i$ near.

The force contribution from the strip $\mathrm{dA}=\mathrm{Bdz}$ in Fig. 1 is $\mathrm{dF}=\sigma \mathrm{Bdz}=$ $=s(1-\omega) \mathrm{Bdz}$. The load induces a moment $\mathrm{M}=\mathrm{F} \cdot \mathrm{e}$ and the equilibrium requirements are

$$
F=\int_{-c}^{c} d F \text { and } M=F \cdot e=\int_{-c}^{c} z d F .
$$

Expressing the load as the ratio of nominal section stress to uniaxial tensile strength and the eccentricity as $\rho=e / r_{i}$ they become

$$
\left.\begin{array}{rl}
\frac{\sigma_{\text {nom }}}{\sigma_{t}}=\frac{F / A}{\sigma_{t}}=\frac{2}{\rho^{2}}\left(1-\frac{\bar{s}}{s_{t}}\right)\left\{\left[\left(1-\rho^{2}\right)\left(1-\frac{\bar{s}}{s_{t}}\right)^{2}+\rho^{2}\right]^{1 / 2}-\left(1-\frac{\bar{s}}{s_{t}}\right)\right\} \text { and } \\
\frac{\Delta s}{s_{t}}=\frac{c}{\rho r_{i}}\left\{\left[\left(1-\rho^{2}\right)\left(1-\frac{\bar{s}}{s_{t}}\right)^{2}+\rho^{2}\right]^{1 / 2}-\left(1-\frac{\bar{s}}{s_{t}}\right)\right\} .
\end{array}\right\}
$$

They give the load as a function of the complementary monotonic loading parameter, $F(\bar{s})$, and $\Delta s(\bar{s})$.

The specimen fails when $\mathrm{dF} / \mathrm{ds}=0$ at $\overline{\mathrm{s}}=\overline{\mathrm{s}}_{1}$ and $\mathrm{F}\left(\overline{\mathrm{s}}_{1}\right)$ is a maximum, that is when

$$
\bar{s}_{1} / s_{t}=1-\left[\frac{\rho}{2(1+\rho)}\right]^{1 / 2} \text { and } \Delta s_{1} / s_{t}=\left(c / r_{i}\right)\left[\frac{\rho}{2(1+\rho)}\right]^{1 / 2} \text {. }
$$


Thus the reduced apparent strength follows as

$$
\sigma_{\text {nom }}^{\mathrm{f}} / \sigma_{t}=1 /\left(1+e / r_{i}\right) \text {. }
$$

For a doubly symmetric cross section $1<c / r_{i}<\sqrt{12}$. If $r_{i}$ is independent of bending axis then the load needn't be applied on a principal axis. Since $c / r_{i}>1$ the apparent strength decreases less for a damaging material than for a non-damaging one, compare Eqs. 13 and 1 . For a solid circular section $c / r_{i}=2$ and the result is $1 /(1+2 \mathrm{e} / \mathrm{c})$ for a damaging material as compared with $1 /(1+4 \mathrm{e} / \mathrm{c})$ for a non-damaging one.

The maximum of $\sigma(z)$ in Eq. 9 at failure equals $\sigma_{t}$ and lies at $z=r_{i}<c$. Thus the tensile extreme fiber stress $\sigma(c)$ is already decreasing, having been $\sigma_{t}$ at some previous point in the load history $\mathrm{F}(\mathrm{s})$. For a non-damaging material $\sigma(z)$ at failure is obviously linear with $\sigma(c)=\sigma_{t}$. The weakening of the damaging material redistributes the stresses over the section so that more of it sustains heavy loads. This explains why a damaging material is less sensitive to eccentric loading than a non-damaging one. The parallel to plastic flow lies close at hand.

Normally resistance strain gauges are used to measure the eccentricity e in a given test. Whether the material is damaging or not one can obtain $\bar{s}$ and $\Delta s$ from suitably positioned gauges. Denote the ratio $\Delta s / \bar{s}$ at failure by $\eta$ and regard it as a measured or known quantity. Then the predicted eccentricity for a damaging material will be

$$
\rho=e / r_{i}=2 \eta^{2} /\left[\left(c / r_{i}\right)^{2}+2 \eta c / r_{i}-\eta^{2}\right],
$$

for a non-damaging one $\rho=\eta r_{i} / c$. The apparent strength predictions become

$$
\sigma_{\text {nom }}^{\mathrm{f}} / \sigma_{t}=1-2 \eta^{2} /\left(\mathrm{c} / \mathrm{r}_{i}+\eta\right)^{2}
$$

and $1 /(1+\eta)$ respectively. The corresponding strength reductions are obviously $O\left(n^{2}\right)$ and $O(n)$ for small $\eta$, they differ by an order of magnitude:
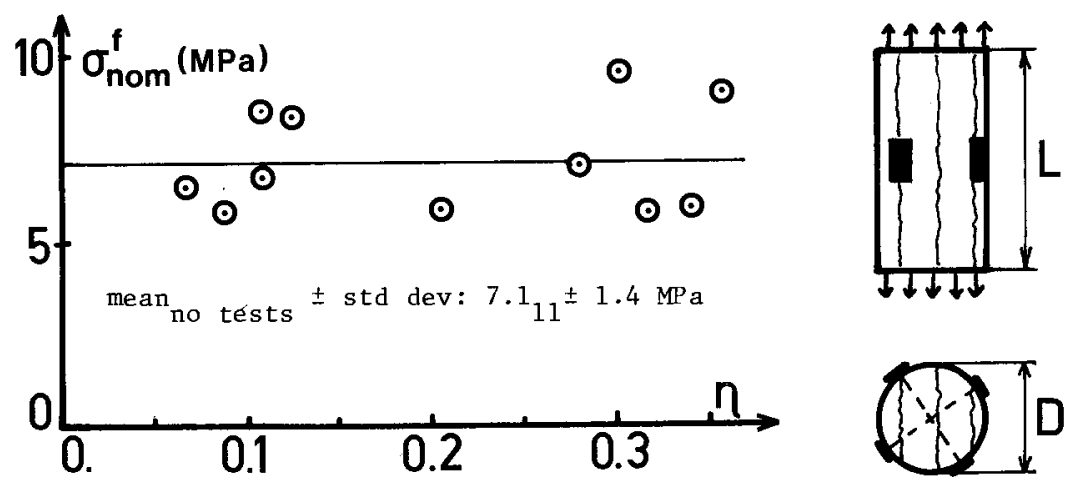

Fig. 2 Apparent uniaxial tensile strength of cylindrical core specimens of Klinthagen limestone. $\mathrm{D}=38-41 \mathrm{~mm}$ and $\mathrm{L} / \mathrm{D}>2$. Four symmetrically arranged strain gauges were mounted on each specimen. 
Take the tests made by Mäki (1982) as an example, see Fig. 2. The largest strain ratio was $\eta \approx 0.35$ which for a non-damaging material would be interpreted as e/c $\approx 0.09$ and an apparent strength of $0.74 \sigma$. For a damaging one there results $\mathrm{e} / \mathrm{c} \approx 0.02$ and $0.96 \sigma_{\mathrm{t}}$. Since the limited data shows no significant strength reduction they support the damaging approach.

\section{CDM IN PURE BENDING}

Consider the same specimen as before but subjected to pure bending, $\mathrm{M}=\mathrm{F} \cdot \mathrm{e}$ with $F \rightarrow 0$ this time. $\varepsilon(z)$ and $s(z)$ will again be linear. The cross section will have to support both tensile and compressive stresses. Denote the neutral axis by $z=-c_{n}$ and the point where the initial damage closes up by $z=-c_{0}$. Geometrical proportionality requires that $s / \Delta s=c_{n} / c$. Here we replace ${ }^{\circ}-\bar{s}_{\text {by }} c_{n}$ and use $s(z)=\Delta s \cdot\left(z+c_{n}\right) / c$ instead of Eq. 8. See ${ }^{n}$ Fig. 3 .

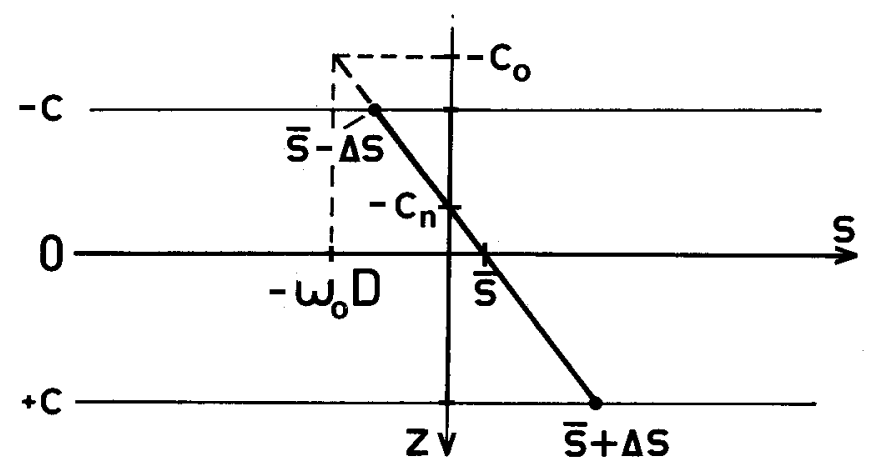

Fig. 3 Net stress distribution $\mathrm{s}(\mathrm{z})$ over cross section $-c \leq z \leq \mathrm{c}$ in pure bending, when damage closure is incomplete, that is when $\bar{s}-\Delta s>-\omega_{0} D$ or $-c_{0}<-c$.

For $\omega_{0}=0$ complete damage closure will occur already on the neutral axis and for $\omega_{0}^{\circ}$ sufficiently large the compressive stresses fail to close the damage completely such that $c_{0}>c$. These two cases will be investigated separately.

We express the moment as the ratio of apparent linear elastic tensile extreme fiber stress to uniaxial tensile strength, $\sigma_{b} / \sigma_{t}$. As long as complete damage
closure doesn't occur Eqs. 10 yield

$$
\left.\begin{array}{c}
\frac{\sigma_{b}}{\sigma_{t}}=\frac{M /(I / c)}{\sigma_{t}}=2 \frac{\Delta s}{s_{t}}\left[1-\left(\frac{r_{i}}{c}\right)^{2}\left(\frac{\Delta s}{s_{t}}\right)^{2}\right]^{1 / 2} \text { and } \\
\frac{\vec{s}}{\Delta s}=\frac{c}{c}=\frac{s_{t}}{\Delta s}\left\{1-\left[1-\left(\frac{r_{i}}{c}\right)^{2}\left(\frac{\Delta s}{s_{t}}\right)^{2}\right]^{1 / 2}\right\},
\end{array}\right\}
$$

with $F=0$. This time the complementary monotonic loading parameter is $\Delta s$. The specimen looses its load carrying capacity when

$$
\Delta s_{1} / s_{t}=\left(c / r_{i}\right) / \sqrt{2} \text { and } c_{n 1} / c=(\sqrt{2}-1)\left(r_{i} / c\right) .
$$


Eqs. 9 and 13 with $\rho \rightarrow \infty$ yield $\sigma(z)$ at failure. Again max $\sigma=\sigma_{t}$ at $z=r_{i}$.

Thus the rupture factor in bending for a specimen of damaging material is

$$
R=\sigma_{t b} / \sigma_{t}=c / r_{i}>1
$$

when $c \geq c$. Eq. 18 predicts that $R=2$ for a solid circular section and that $R=\sqrt{3} \approx 1.7$ for a rectangular one. Experimental values (Ouchterlony, 1981) for Bohus granite, 2.1 and 1.7 respectively, and Ekeberg marble, $2.3-2.4$ and 2.0 respectively, aren't far off.

For a material with complete damage closure under compressive stresses the equilibrium integrals are separated into compressive and tensile parts. In this case $c=c$. Introduce the dimensionless variables $\zeta=z / c$, the breadth function ${ }^{n} \mathrm{~b}(\zeta)^{\circ}=\mathrm{B}(\mathrm{z}) / \mathrm{B}$ so that $\mathrm{b}(0)=1$, and $\zeta_{\mathrm{O}}=\mathrm{c}_{\mathrm{o}} / \mathrm{c}$. Then Eqs. 10 reduce to.

$$
\left.\begin{array}{l}
\frac{\sigma_{b}}{\sigma_{t}}=\frac{4 A}{B c} \cdot \frac{\zeta_{o}}{J_{2}\left(\zeta_{0}\right)}\left\{1+\zeta_{0}^{2}\left(\frac{c}{r_{i}}\right)^{2}\left[1-\frac{J_{3}\left(\zeta_{0}\right)}{\zeta_{0} J_{2}\left(\zeta_{o}\right)}\right]\right\} \text { and } \\
\frac{\Delta s}{s_{t}}=\frac{2 A}{B c} \zeta_{o} / J_{2}\left(\zeta_{0}\right) \text { where } J_{n}\left(\zeta_{0}\right)=\int_{\zeta_{0}}^{1}\left(\zeta+\zeta_{0}\right) n_{b}(\zeta) d \zeta .
\end{array}\right\}
$$
A sufficient condition for $R$ being the maximum of $\sigma_{b} / \sigma_{t}$ at $\zeta_{\mathrm{o}}$ is that $\Delta_{\mathrm{s}}\left(\zeta_{\mathrm{o}}\right)$
has an inverse in the relevant interval. This will obvious 1 y depend on the explicit forms of $J_{n}\left(\zeta_{0}\right)$.

Take the rectangular section first (Krajcinnovic, 1979). For it $A=2 B c$, $\mathrm{c} / \mathrm{r}_{\mathrm{i}}=\sqrt{3}, \mathrm{~b}(\zeta)=\frac{1}{1}$, and $\mathrm{J}_{\mathrm{n}}\left(\zeta_{0}\right)=\left(1+\zeta_{0}\right)^{\mathrm{n} \neq l} /(\mathrm{n}+1)$. Thus we obtain

$$
\left.\begin{array}{rl}
\sigma_{\mathrm{b}} / \sigma_{\mathrm{t}} & =6\left(4 \zeta_{\mathrm{o}}-9 \zeta_{\mathrm{o}}^{2}+35 \zeta_{\mathrm{o}}^{3}\right) /\left(1+\zeta_{\mathrm{o}}\right)^{3} \text { and } \\
\Delta \mathrm{s} / \mathrm{s}_{\mathrm{t}} & =12 \zeta_{\mathrm{o}} /\left(1+\zeta_{\mathrm{o}}\right)^{3} .
\end{array}\right\}
$$

Since $\zeta_{\mathrm{ol}}=(13-\sqrt{9} 7) / 18 \approx 0.175$ and $\Delta \mathrm{s} / \mathrm{s}$ increases monotonically until $\zeta_{\mathrm{f}}=0.5$ then $R \stackrel{\circ}{=} 1$ 1.63. This agrees with the result of Bert and Kumar (1980) who gefine strength failure differently than Krajcinovic (1979).

The solid circular section is more important for rock. For it $\mathrm{A} / \mathrm{BC}=\pi / 2$, $\mathrm{c} / \mathrm{r}_{\mathrm{i}}=-\frac{1}{2}$, and $\mathrm{b}(\bar{\zeta})=\sqrt{\left(1-\bar{\zeta}^{2}\right)}$. In this case $\zeta_{\mathrm{l}}=0.149$ with $\Delta \mathrm{s} / \mathrm{s}_{\mathrm{t}}$ still increasing monotonically. Hence the rupture factor becomes $R=1.85$.

Unfortunately complete damage closure under compressive stresses lowers the predicted $R$-values, but not much. Thus an analysis of the intermediate cases $c_{n}<c_{0}<c$ wasn't made. The damage law $\omega(s)$ can probably be chosen so that theory and experiments agree but that choice should have a sounder physical basis.

The results for pure bending also apply to three point bending, a point loaded cantilever, and four point bending, all cases with statically determinate moment distributions. For them the damaging material will redistribute the stress over the cross section but not from one section to another.

\section{CONCLUSIONS}

CDM permits a unified approach to both eccentric uniaxial loading and pure bending of strength test specimens of rock. The damaging implies a realistic 
deviation from linearly elastic material behavior which changes the conventional prediction formulas for apparent strength. The damage law used here is simple. Hence these CDM results should only be regarded as qualitative, yet they are quite reasonable when compared with experiments.

Several multiaxial CDM formulations exist (Chaboche, 1981). Thus a unified approach to all common strength tests for rock is possible, not only multiaxial tensile ones like the hydraulic tension test, the Brazilian disc test, and the point load test. CDM even connects the uniaxial compression test with $\sigma$ (Krajcinovic and Fonseka, 1981).

\section{REFERENCES}

Bert, C. W., and M. Kumar (1980). Discussion of Krajcinovic (1979). J. Appl. Mechs., 47, 449-450.

Chaboche, J.-L. (1981). Continous damage mechanics - A tool to describe phenomena before crack initiation. Nucl. Engng. Des., 64, 233-247.

Grady, D. E. and M. E. Kipp (1980). Continuum modelling of explosive fracture in oil shale. Int. J. Rock Mechs. Min. Sci., 17, 147-157.

Hult, J. (1979). CDM - Capabilities, limitations and promises. In proc. interdisciplinary conf. Mechanisms of Deformation and Fracture, ed. K. Easterling, Pergamon Press 233-247.

Jansson, J. (1978). Crack in Material with Damage Formation - A Mechanical Analysis. Diss. Chalmers Institute of Technology, Gothenburg, Sweden. Krajcinovic, D. (1979). Distributed damage theory of beams in pure bending.
J. Appl. Mechs., 46, 592-596.

Krajcinovic, D. and G. U. Fonseka (1981). The continuous damage theory of brittle materials. In 2 parts. J. Appl. Mechs., 48, 809-824.

Mäki, K. (1982). Personal communication.

Ouchterlony, F. (1981). A distributed damage approach to combined bending and axial loading of rock beams. Swedish Detonic Research Foundation report DS 1981:19, Stockholm, Sweden.

Timoshenko, S. (1940). Strength of Materials Part I - Elementary Theory and Problems, 2nd ed. Van Nostrand, Toronto. Chap. 51.

Vutukuri, V. S., R. D. Lama, and S. S. Saluja (1974). Handbook on Mechanical Properties of Rock, Vol. 1. Trans Tech Publications, Aedermansdorf, Switzerland. Chap. 3. 


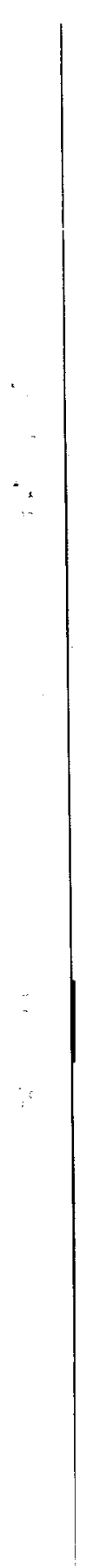

